Research Paper

\title{
The correlation between NEDD4L and HIF-1 $\alpha$ levels as a gastric cancer prognostic marker
}

\author{
Xingwang Jiang ${ }^{1 *}$, Shangxin Zhang ${ }^{1 *}$, Zihuan Yin², Yi Sheng ${ }^{1}$, Qiang Yan ${ }^{1}$, Ruochuan Sun ${ }^{1}$, Mingdian Lu1, \\ Zhen Zhang1, Yongxiang $\mathrm{Li}^{1}{ }^{\boxplus}$ \\ 1. Department of General Surgery, First Affiliated Hospital of Anhui Medical University, Hefei 230022, People's Republic of China \\ 2. Department of Thoracic Surgery, Anhui chest hospital, Hefei 230022, People's Republic of China. \\ *Xingwang Jiang and Shangxin Zhang equally contributed to this work. \\ $\bowtie$ Corresponding author: E-mail: liyongxiang@ahmu.edu.cn; Tel.: +86-0551-62923887
}

(1) The author(s). This is an open access article distributed under the terms of the Creative Commons Attribution License (https://creativecommons.org/licenses/by/4.0/). See http://ivyspring.com/terms for full terms and conditions.

Received: 2019.03.05; Accepted: 2019.09.09; Published: 2019.10.21

\begin{abstract}
NEDD4L (neural precursor cell expressed developmentally down-regulated 4-like) protein is a member of ubiquitin ligases Nedd4 family. Although studies have shown that Nedd4L may act as a tumor suppressor in various cancers, including gastric cancer (GC), its clinical significance and the diagnostic value in GC is not well defined. HIF-1a (hypoxia inducible factor family of transcription factors) is actively involved in the metabolism of many tumors, although the relationship between its expression levels and clinical significance in GC still need to be established. In this study, the level of HIF-1 $\alpha$ and NEDD4L mRNA and protein in 25 freshly frozen GCand matched normal-tissues were determined by western blot and quantitative PCR (qPCR). Additionally, immunohistochemistry assay was performed to measure the protein level of NEDD4L and HIF-1a in 124 GC and 25 normal control tissues. We observed that the NEDD4L mRNA and protein levels decreased significantly $(P<0.001)$ in GC tissues, while that of HIF-1 $\alpha$ increased $(P<0.001)$, and they both were associated with a poor prognosis, as was the case in patients with lower NEDD4L and higher HIF-1 $\alpha$ expression $(P<$ $0.001)$. On correlation analysis, a significantly negative relationship $(r=0.288, P<0.01)$ was revealed between NEDD4L and HIF-1 $\alpha$ expressions. Multivariate analysis revealed that co-expression of NEDD4L $(P<0.05)$ and HIF-1a $(\mathrm{P}<0.001)$ were independent predictors of GC prognosis. Thus, the correlation of NEDD4L and HIF-la levels may act as a prognostic marker of GC.
\end{abstract}

Key words: gastric cancer, NEDD4L, HIF-1a, prognosis, co-expression

\section{Introduction}

Throughout the world, a major cause of deaths due to cancer is because of gastric cancer (GC), amounting to $20 \%$ of the total world population leading to a huge burden on health and economy[1]. In 2012 alone, nearly 720000 patients lost their lives due to gastric cancer and, more than 950000 new diagnoses were made each year [2]. The occurrence and development of GC is complex, multi-factorial, and involves several genes. In several cases, the disease is inoperable during diagnosis or may recur even after curative resection. Therefore, it becomes essential to determine a biomarker that is prognostic for the development and progression of GC, to enable an improved and accurate prediction of its recurrence.

Over recent years, NEDD4L has been shown to play key roles in the prognosis of tumors such as in prostate cancer[3], non-small cell lung cancer (NSCLC)[4], cancer of gallbladder[5], gastric cancer [6], malignant glioma[7], and colorectal cancer[8]. However, NEDD4L has been observed to have contradictory roles, as it promotes cancer of gallbladder cancer and malignant glioma, and has a protective role in colorectal and gastric cancer. NEDD4L is closest in homology to and shares similar E2 specificities with NEDD4, an evolutionarily conserved E3 ligase and a prototype protein like the ubiquitin ligases from NEDD4 family. It was first identified as a down-regulated gene in the course of development of the central nervous system of an early embryo. The members of NEDD4 family have a distinct modular domain architecture with domain for $\mathrm{Ca} 2+$ phospholipid binding (C2) at the amino 
terminal, the 2-4 WW domains for protein to protein interactions and the HECT domain at the carboxyl terminus[9]. The role of NEDD4L in the kidney as the inhibitor of the epithelial sodium channel $(\mathrm{ENaC})$ is well-known[10]. NEDD4L also regulates the PI3KAKT signaling pathway via PIK3CA ubiquitination. Although the NEDD4L expression has been evaluated in the prognosis of GC, a more elaborate study on a larger sample size is required to highlight its specific mechanism and clinical significance.

HIF-1 (hypoxia inducible factor), a transcription factor, is a heterodimer of the HIF-1 $\alpha$ and HIF-1 $\beta$ subunits[11] and has a key role in tumor cells for energy production for maintaining their metabolism [12]. Normally, HIF-1a undergoes quick, proteasomemediated degradation, but under hypoxic conditions, it is stabilized. It is overexpressed in various cancers, including that of the ovary, breast, uterus, cervix, and oropharynx and its overexpression often positively leads to poor prognosis[13-16]. HIF-1a is favorably linked to phosphorylated AKT. Studies have also shown that promotion of the AKT-HIF-1a-VEGF pathway, independent of hypoxia, aids in GC tumorigenesis and angiogenesis [17]. While enhanced expression of HIF1a is often linked to poor prognosis in GC [18], nevertheless, some conflicting prognostic data is still reported $[19,20]$.

In GC, the PI3K-AKT-mTOR pathway may activate HIF-1a [17]. Interestingly, NEDD4L regulates the PI3K-AKT signaling pathway via ubiquitination of PIK3CA [21]. We hypothesized that the NEDD4L and HIF-1a co-expression plays an important part in clinical prognosis in GC. Therefore, in this study, we explored the HIF-1a and NEDD4L protein expressions in on a larger number of human GC tissues, their correlation and effect on the GC patient prognosis.

\section{Materials and Methods}

\section{Extraction of total RNA and quantitative real-time PCR}

Fresh frozen human GC tissue were used for extraction of total RNA using TRIzol Reagent from Invitrogen (USA), and reverse transcription was carried out using the ReverTra Ace qPCR RT Kit from Toyobo (Japan) as per instructions of the manufacturer. The NEDD4L and HIF-1a mRNA levels were determined by RT-PCR on the Sequence Detection System ABI 7900HT from Applied Biosystem (USA) and using SYBR-Green dye from Toyobo (Japan), and the following primers: NEDD4L, sense (5'- TCCAATGGTCCTCAGCTGTTTA -3') and antisense (5'-ATTTTCCACGGCCATGAGA-3'); HIF1a, sense (5'-ATCCATGTGACCATGAGGAAATG-3') and antisense (5'-TCGGCTAGTTAGGGTACACTTC3'); GAPDH, sense (5'-ATCAAGAAGGTGGTGAAGC AGG-3'), antisense (5'-CGTCAAAGGTGGAGGAGT GG-3'). The $2^{-\Delta{ }^{\prime} \mathrm{CT}}$ method was used to estimate the fold changes in expression.

\section{Clinical specimens}

The department of general surgery in the First Affiliated Hospital of Anhui Medical University (Hefei, China) provided us with human GC tissue microarrays (TMA) of 124 primary GC and 25 normal gastric tissues that were randomly selected were obtained from between December 2006 and September 2008 and the follow-up period was from 2006 to 2013. No prior radio-, chemo-, or other tumor-specific therapy was given to any of the patients before the sampling. The time from the day 1 post-operation to the day of death was defined as the overall survival time (OS). Samples were collected afresh from $5 \mathrm{~cm}$ from the edge of the tumor and from corresponding normal tissues, dipped in liquid nitrogen for quick freeze, and kept at $-80^{\circ} \mathrm{C}$ until RNA and protein were extracted. Two experienced pathologists examined the result of immunohistochemical staining. For TNM staging, the standard was the seventh edition of TNM Grading Standard specified by the American Joint Committee on Cancer (AJCC). All patients signed a written and informed consent form. The Institute Research Ethics Committee of Anhui Medical University's First Affiliated Hospital reviewed and approved this protocol.

\section{Western blotting}

The RIPA buffer containing a mixture of protease inhibitor was added to the fresh-frozen tissues to extract total protein and their quantification was done using BCA Protein Assay Kit from Beyotime (China). Protein from the samples were separated on SDS polyacrylamide gel $(10 \%)$ by electrophoresis and transferred to nitrocellulose membrane from Millipore (USA). After blocking for 1 $\mathrm{h}$ with nonfat milk (5\% in tris-buffered saline with tween-20) solution at room temperature, anti-NEDD4L rabbit antibody (1:1000; Proteintech, China) and anti-HIF-1A rabbit antibody (1:1000; Abcam, USA) were added to the membrane and kept overnight at $4^{\circ} \mathrm{C}$. Then, the respective secondary antibody (1:10000, Proteintech, China) were added to the membrane and after $1 \mathrm{~h}$ incubation at room temperature, enhanced chemiluminescence system was used to detect the immunoreactive signals. The internal control in this assay was GAPDH. 


\section{Immunohistochemical staining and evaluation}

A conventional immunohistochemical (IHC) staining protocol was used. Tissues from GC and healthy samples were fixed in formalin-fixed, paraffin- embedded, and used to prepare TMA. The TMA section was kept in xylene for removing paraffin, rehydrated in alcohol at different concentrations, then treated with $3 \% \mathrm{H} 2 \mathrm{O} 2$ and subsequently microwaved in the citrate buffer (10 $\mathrm{mM}, \mathrm{pH}$ 6.0) for $5 \mathrm{~min}$ at $120^{\circ} \mathrm{C}$. The sections were blocked with BSA (bovine serum albumin, 1\%) for 0.5 $h$, incubated overnight with anti-NEDD4L antibody (1: 100; proteintech), anti-HIF-1a (1: 300; Abcam) at 4 ${ }^{\circ} \mathrm{C}$, and then with a secondary antibody labeled with peroxidase. The slides were then rated to assess the level of protein expression on the basis of the intensity of staining of the product. Scores were defined as: 0 , no staining; 1 , weak staining; 2 , moderate staining; 3 , strong staining) and percent of positive tumor cells were scored as: 0 , none; $1,1 \%-25 \% ; 2,25 \%-75 \% ; 3,>$ $75 \%$. The range of the final score was 0 and 9 , defined as negative or weak $(-, 0 \sim 2)$, and positive $(+, 3 \sim 9)$.

\section{Statistical analysis}

The software SPSS 17.0 (SPSS Inc., USA) and GraphPad Prism 5 (San Diego, CA) were used for

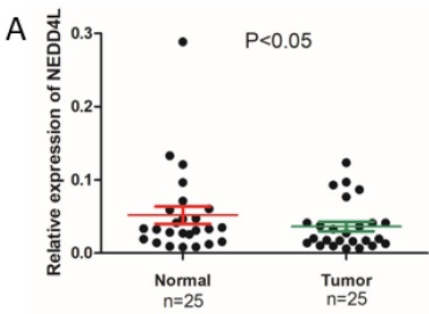

B

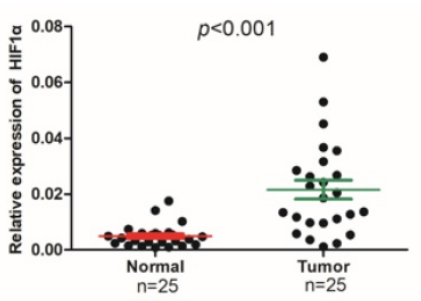

C

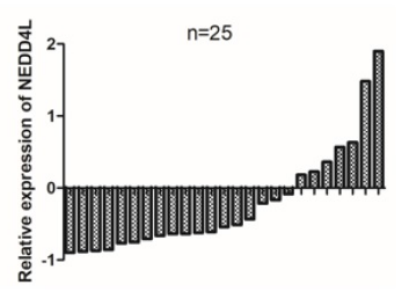

D

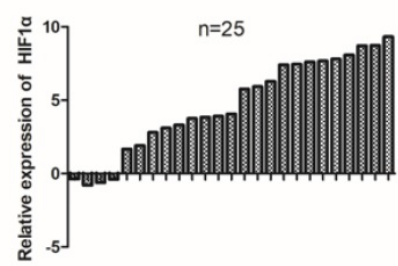

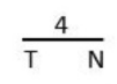
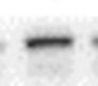

HIF-1 $\alpha$

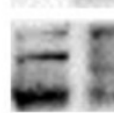

GAPDH

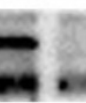
2

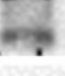

Figure 1.The mRNA and protein expression level of NEDD4L and HIF1a in clinical samples. Scatter plots of the relative expression of NEDD4L (A) and HIF-1a (B) mRNA in tumor and normal tissues. Bar plots of NEDD4L (C) and HIF-1 $\alpha(D)$ expression in GC tissues compared with corresponding normal tissues. In each patient was presented as the log2 ratio of tumor tissue/normal tissue. $(\mathrm{E})$ The protein expression level of NEDD4L and HIF-1a were analyzed by western blot assay.Representative protein expression level of NEDD4L and HIF-1 $\alpha$ in 6 pairs of tumor (T) and corresponding normal tissues $(N)$. statistical analyses. For analyses of NEDD4L and HIF-1a levels and clinicopathological factors, the Chi-square test was applied. The correlation between NEDD4L and HIF-1a were done by Spearman's rank test. The dissimilarities between gastric and non-tumor tissues in terms of the levels of HIF-1a and NEDD4L were compared using Student's t-test (two-tailed). For univariate and multivariate analysis, the model of Cox's proportional hazards was used to spot independent prognostic factors. The statistically significant values were $\mathrm{P}<0.05$.

\section{Results}

\section{NEDD4L and HIF-1 $\alpha$ expressions in fresh GC tissue}

The qPCR was carried out to evaluate the NEDD4L and HIF-1a mRNA expressions in fresh GCand corresponding normal-tissues. As per the Figure 1, the expressions of NEDD4L in GC tissues was notably down-regulated, whereas that of HIF-1a was upregulated. We carried out western blot to examine the association between levels of NEDD4L and HIF-1a in relation to their respective mRNA levels, and found that while NEDD4L expression reduced distinctly in GC tissues, the HIF-1a increased notably, which is in accordance with the qPCR result (Figure 1).

\section{Immunohistochemical staining for NEDD4L and HIF-1 a levels in matched GC and normal tissues}

To assess the NEDD4L and HIF-1a expression levels, the TMA that harbored 124 GC and 25 normal gastric tissues was detected by immunohistochemical staining. The staining results reveal the NEDD4L expression mainly in the cytoplasm and while $45.97 \%(57 / 124)$ in GC tissues stained positively for NEDD4L, the value was $72 \%(18 / 25)$ for the normal gastric tissues $(\mathrm{P}=0.018)$. Moreover, while HIF-1a expressed mainly in nuclear region, and $66.13 \%$ $(82 / 124)$ of gastric cancer tissues stained positive, and the value was $32 \%(8 / 25)$ in normal tissues $(\mathrm{P}=0.001)$ (Figure 2). These results were in accordance with that of the western blot results. These results indicate that the NEDD4L expression is low in gastric cancer, whereas HIF-1a is overexpressed. 

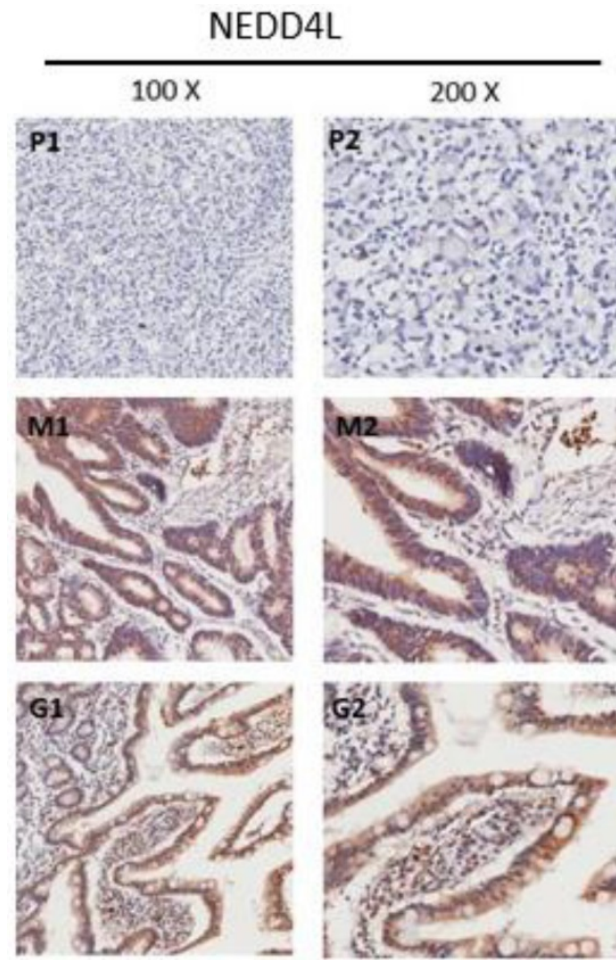

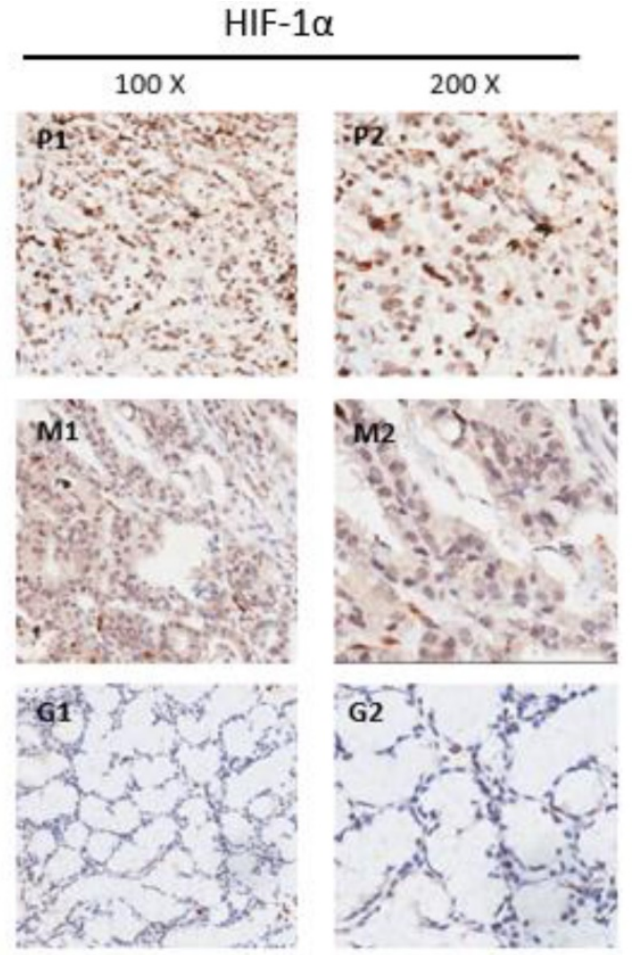

Figure 2. Immunohistochemical staining of NEDD4L, HIF-1 $\alpha$ protein in GC and normal gastric tissues. Representative images of NEDD4L and HIF-1 $\alpha$ as followings: P1, P2 (poor differentiated ) and M1, M2 (moderately differentiated) staining in GC, G1,G2 staining in normal gastric tissue. Magnification: 100× (P1, M1 and G1) and 200× (P2,M2) and G2).

\section{Clinical significance and the correlation of NEDD4L and HIF-1 $\alpha$ expression in GC}

The Table 1 lists the clinicopathological characters of NEDD4L and HIF-1a. The low expression of NEDD4L significantly correlated with tumor invasion $(P=0.025)$, tumor differentiation $(P=0.039)$ and TNM staging $(\mathrm{P}=0.03)$. Likewise, enhanced HIF-1a expression correlated with tumor size $(\mathrm{P}=0.049)$, TNM staging $(\mathrm{P}=0.014)$ and depth of tumor invasion $(P=0.004)$. Contrarily, the NEDD4L and HIF-1a expression did not correlate with gender, age, lymph node metastasis and tumor location. As shown in Figure 3 and table 3, the expression of NEDD4L in GC tissues correlated negatively with HIF-1a expression $(\mathrm{r}=-0.288, \mathrm{P}=0.001)$.

\section{Prognostic Significance of NEDD4L and HIF-1 $\alpha$ in GC Patient Survival}

We performed survival analysis of 124 patients using clinical follow-up results to evaluate the prognostic potential of NEDD4L and HIF-1a in GC and the results are presented in figure 4 and table 2 . The patient cumulative survival rate for 3-year period with negative and positive NEDD4L expressions were $41.8 \%$ and $77.2 \%$, respectively. Likewise, the patient cumulative survival rate for 5-year period with negative and positive expression of NEDD4L were $35.6 \%$ and $70.2 \%$, respectively. Thus, patients with negative NEDD4L expression had a significantly worse $(\mathrm{P}<0.001)$ prognosis. However, the 3- and 5year cumulative survival rate of HIF-1a negative patients were $85.5 \%$ and $75.8 \%$, respectively, which were notably higher than that of the survival rates of HIF-1a positive patients $(30.6 \%$ and $27.2 \%$, respectively). Apparently, high expression of HIF-1a was linked to poor prognosis for GC patients $(\mathrm{P}<0.001)$.

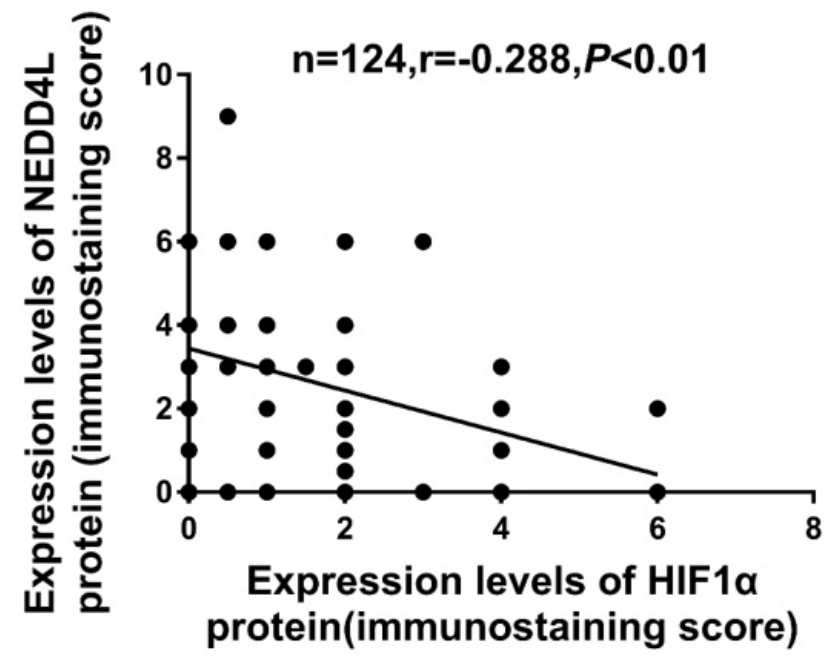

Figure 3. Correlation between NEDD4L and HIF-1 $\alpha$ in GC tissues.

We then explored the relationship between different combinations of NEDD4L and HIF-1a expressions and the prognosis of GC patients. Based on NEDD4L and HIF-1a expressions, the patients 
were divided into four groups: (1), NEDD4L-/HIF$1 a+;$ (2), NEDD4L+/HIF-1a-; (3), NEDD4L-/HIF-1a-; (4), NEDD4L+/ HIF-1 ++ . Among the four groups, the worst prognosis was observed in NEDD4L-/ HIF-1a+ patients (Figure 4; mean survival time, $26.538 \pm 3.530$ months), whereas the best prognosis was observed in the NEDD4L+/HIF-1a- patients (mean survival time, $66.528 \pm 2.550$ months). While there was a significant difference between these two groups concerning OS $(\mathrm{P}<0.001)$, no such difference in OS was observed between NEDD4L+/HIF-1a+ and NEDD4L-/HIF-1agroups $(p=0.07)$, thus, indicating that a high level of NEDD4L or a low level of HIF-1a may functionally compensate for each other's effects in the prognosis of GC patients.

Table 1.Relationship between NEDD4L and HIF-1 $\alpha$ expression and clinicopathological variables $(n=124)$

\begin{tabular}{|c|c|c|c|c|c|c|c|}
\hline \multirow[b]{2}{*}{ Variables } & \multirow[b]{2}{*}{ Total } & \multicolumn{2}{|c|}{$\begin{array}{l}\text { NEDD4L } \\
\text { expression }\end{array}$} & \multirow{2}{*}{$\begin{array}{l}P \\
\text { value }\end{array}$} & \multicolumn{2}{|c|}{$\begin{array}{l}\text { HIF-1a } \\
\text { expression }\end{array}$} & \multirow{2}{*}{$\begin{array}{l}P \\
\text { value }\end{array}$} \\
\hline & & negative & positive & & negative & positive & \\
\hline Gender & & & & 0.670 & & & 0.826 \\
\hline Male & 96 & 43 & 53 & & 33 & 63 & \\
\hline Female & 28 & 14 & 14 & & 9 & 19 & \\
\hline Age at surgery ( & & & & 1 & & & 0.312 \\
\hline$<61$ & 61 & 33 & 28 & & 18 & 43 & \\
\hline$\geq 61$ & 63 & 29 & 34 & & 24 & 39 & \\
\hline Tumor size $(\mathrm{cm})$ & & & & 0.448 & & & 0.049 \\
\hline$<6$ & 41 & 42 & 40 & & 9 & 32 & \\
\hline$\geq 6$ & 83 & 25 & 17 & & 33 & 50 & \\
\hline Differentiation & & & & 0.039 & & & 0.349 \\
\hline High/moderate & 32 & 12 & 20 & & 13 & 19 & \\
\hline poor/no & 92 & 55 & 37 & & 29 & 63 & \\
\hline Location & & & & 0.975 & & & 0.854 \\
\hline Cardia & 64 & 34 & 30 & & 22 & 42 & \\
\hline Corpus & 27 & 15 & 12 & & 8 & 19 & \\
\hline antrum & 33 & 18 & 15 & & 12 & 21 & \\
\hline T stage & & & & 0.025 & & & 0.004 \\
\hline $\mathrm{T} 1 / \mathrm{T} 2$ & 26 & 9 & 17 & & 15 & 11 & \\
\hline T3/T4 & 98 & 58 & 40 & & 27 & 71 & \\
\hline N stage & & & & 0.448 & & & 0.097 \\
\hline No & 41 & 47 & 36 & & 18 & 23 & \\
\hline Yes & 83 & 20 & 21 & & 24 & 59 & \\
\hline TNM & & & & 0.030 & & & 0.014 \\
\hline I/II & 52 & 30 & 22 & & 24 & 28 & \\
\hline III/IV & 72 & 45 & 27 & & 18 & 54 & \\
\hline
\end{tabular}

To investigate if NEDD4L and HIF-1a could independently predict the GC prognosis, we carried out Cox's univariate regression analysis and found that the parameters including tumor size, metastasis of the lymph node, depth of invasion, differentiation, TNM stage, and the NEDD4L and HIF-1a levels significantly corresponded to OS in GC patients (Table 2). Multivariate analysis revealed that for overall survival in GC patients, tumor differentiation and the combined expression of NEDD4L and HIF-1a were independent prognostic factors (Table 3).
Table 2. Univariate analysis of the correlation between clinicopathological factors and survival of patients with GC

\begin{tabular}{|c|c|c|c|c|}
\hline \multirow[t]{2}{*}{ Parameters } & \multicolumn{2}{|c|}{ Cumulative Survival Rates, $\%$} & \multirow{2}{*}{$\begin{array}{l}\text { Mean Survival } \\
\text { Time, month }\end{array}$} & \multirow{2}{*}{$\begin{array}{l}P \\
\text { value }\end{array}$} \\
\hline & 3-Year & 5-Year & & \\
\hline Gender & & & & 0.452 \\
\hline Male & 63.5 & 47.8 & 47.4 & \\
\hline Female & 64.3 & 60.3 & 49.5 & \\
\hline Age(y) & & & & 0.633 \\
\hline$<61$ & 62.3 & 53.9 & 48.3 & \\
\hline$\geq 61$ & 60.3 & 49.2 & 47 & \\
\hline \multicolumn{3}{|c|}{ Tumor diameter (cm) } & & 0.016 \\
\hline$<6$ & 46.3 & 39 & 39.5 & \\
\hline$\geq 6$ & 67.5 & 57.8 & 51.9 & \\
\hline Location & & & & 0.148 \\
\hline Cardia & 62.5 & 46.6 & 47.1 & \\
\hline Corpus & 48.1 & 44.4 & 40.8 & \\
\hline antrum & 66.7 & 66.7 & 54.3 & \\
\hline \multicolumn{3}{|l|}{ Depth of invasion } & & 0.000 \\
\hline $\mathrm{T} 1 / \mathrm{T} 2$ & 96.2 & 92.3 & 68.2 & \\
\hline $\mathrm{T} 3 / \mathrm{T} 4$ & 48 & 40.7 & 42.1 & \\
\hline \multicolumn{3}{|c|}{ Histological grade } & & 0.001 \\
\hline Well/moderate & 81.3 & 78 & 62 & \\
\hline Poor/not & 50 & 42.4 & 43 & \\
\hline \multicolumn{3}{|c|}{ Lymph node metastasis } & & 0.000 \\
\hline No & 87.8 & 82.9 & 66.8 & \\
\hline Yes & 43.4 & 34.6 & 38.3 & \\
\hline TNM stage & & & & 0.000 \\
\hline I-II & 88.5 & 84.6 & 67.1 & \\
\hline III-IV & 36.1 & 27.4 & 33.9 & \\
\hline \multicolumn{3}{|c|}{ NEDD4L expression } & & 0.000 \\
\hline negative & 41.8 & 35.6 & 37.7 & \\
\hline positive & 77.2 & 70.2 & 59.1 & \\
\hline \multicolumn{5}{|l|}{ HIF1a expression } \\
\hline negative & 85.5 & 75.8 & 64.4 & 0.000 \\
\hline positive & 30.6 & 27.2 & 30.8 & \\
\hline \multicolumn{3}{|c|}{ NEDD4L/HIF1a expression } & & 0.000 \\
\hline NEDD4L-/HIF1a- & 78.3 & 65.2 & 58.8 & 0.000 \\
\hline NEDD4L+/HIF1a- & 89.7 & 82.1 & 66.5 & \\
\hline NEDD4L-/HIF1a+ & 22.7 & 20.2 & 26.5 & \\
\hline NEDD4L+/HIF1a+ & 50 & 44.4 & 41.4 & \\
\hline NEDD4L+/HIF1a- & 89.7 & 82.1 & 66.5 & 0.000 \\
\hline NEDD4L-/HIF1a+ & 22.7 & 20.2 & 26.5 & \\
\hline NEDD4L-/HIF1a- & 78.3 & 65.2 & 58.8 & 0.070 \\
\hline NEDD4L+/HIF1a+ & 50 & 44.4 & 41.4 & \\
\hline
\end{tabular}

\section{Discussion}

The human gene NEDD4L encodes ubiquitin ligase NEDD4L, which downregulates epithelial sodium channels of kidney, which are associated with essential hypertension[22]. Subsequently, a broader role for this ubiquitin ligase has been reported in several types of tumors, with varying outcomes $[3,7$, 23]. For instance, decreased NEDD4L expression corresponds to an increased prostate cancer risk, while that in NSCLC corresponds with a poor prognosis[4].

In this study, we found that in most GC tissues the NEDD4L mRNA and protein levels were significantly reduced. This too, significantly correlated with tissue differentiation, TNM staging and depth of tumor invasion, significantly shorter survival, as was seen in the subsequent survival analysis, and is in accordance with previous reports. 
Multivariate Cox analysis revealed that NEDD4L was an independent predictor factor of GC.

NEDD4L affects tumor-associated pathways through ubiquitination and plays an important role in tumorigenesis and development. For example, Kuratomi et al and Gao et al. found that NEDD4L inhibited the TGF- $\beta$ signaling pathway by accelerating the ubiquitination of activated Smads and promoting their degradation[24, 25]. NEDD4L also target Dvl2, a key component of Wnt signaling, and negatively regulate Wnt signaling pathways[26]. LoïcBroix et al. reported that NEDD4L dysregulates the AKT-mTOR pathway by disrupting mTORC1mediated signaling, a finding similar to another report showing that NEDD4L catalyzed the PIK3CA ubiquitination and regulated PI3K-AKT signaling[21]. Therefore, NEDD4L may be considered a tumor suppressor, although, this needs to be verified through direct and robust experimental evidence. Our previous experimental results indicate that NEDD4L is associated with tumor differentiation, invasion, and metastasis, and NEDD4L expression is also associated with the prognosis of patients with gastric cancer.

The tumor microenvironment is characterized by nutrient supply diversity, $\mathrm{pH}$, and oxygenation[27]. Particularly, hypoxia is associated with tumor development, progression, metastasis, inadequate response to treatment, and changes in tumor cell behavior through many $\mathrm{O}^{2}$-sensitive pathways. The most studied is HIFs mediation[28, 29]. This NEDD4L ubiquitination is affected by hypoxic stress in tumor tissues; therefore, we hypothesized that NEDD4L might be associated with hypoxia-inducible gene HIF-1a.

HIF-1a mRNA and protein levels were significantly higher in tumor tissues than in normal tissues. The survival analysis also revealed a notably shorter survival time for GC patients with high HIF-1a levels than those with lower expression. Furthermore, increased HIF-1a expression also correlated with tumor size, TNM staging, and depth of tumor invasion. These results corroborated with the multivariate Cox regression analysis and reveal that HIF-1a is a factor for the GC prognosis. Our results indicate that the role of HIF-1a in gastric cancer is the opposite of NEDD4L.

Given the correlation between NEDD4L and HIF-1a, we hypothesized that they may be jointly involved in tumor development and progression. We then explored the link between the expressions of NEDD4L and HIF-1a in GC and ultimately their relationship with patient prognosis. Our results showed an inverse relationship between NEDD4L and HIF-1a expressions $(\mathrm{r}=-0.288, \mathrm{P}<0.01)$. Additionally, we observed the worst prognosis in the NEDD4L- / HIF-1a+ patients, suggesting that the correlation of NEDD4L and HIF-1a is a more credible indicator of GC prognosis, although, this requires further study to determine the specific mechanism involved.
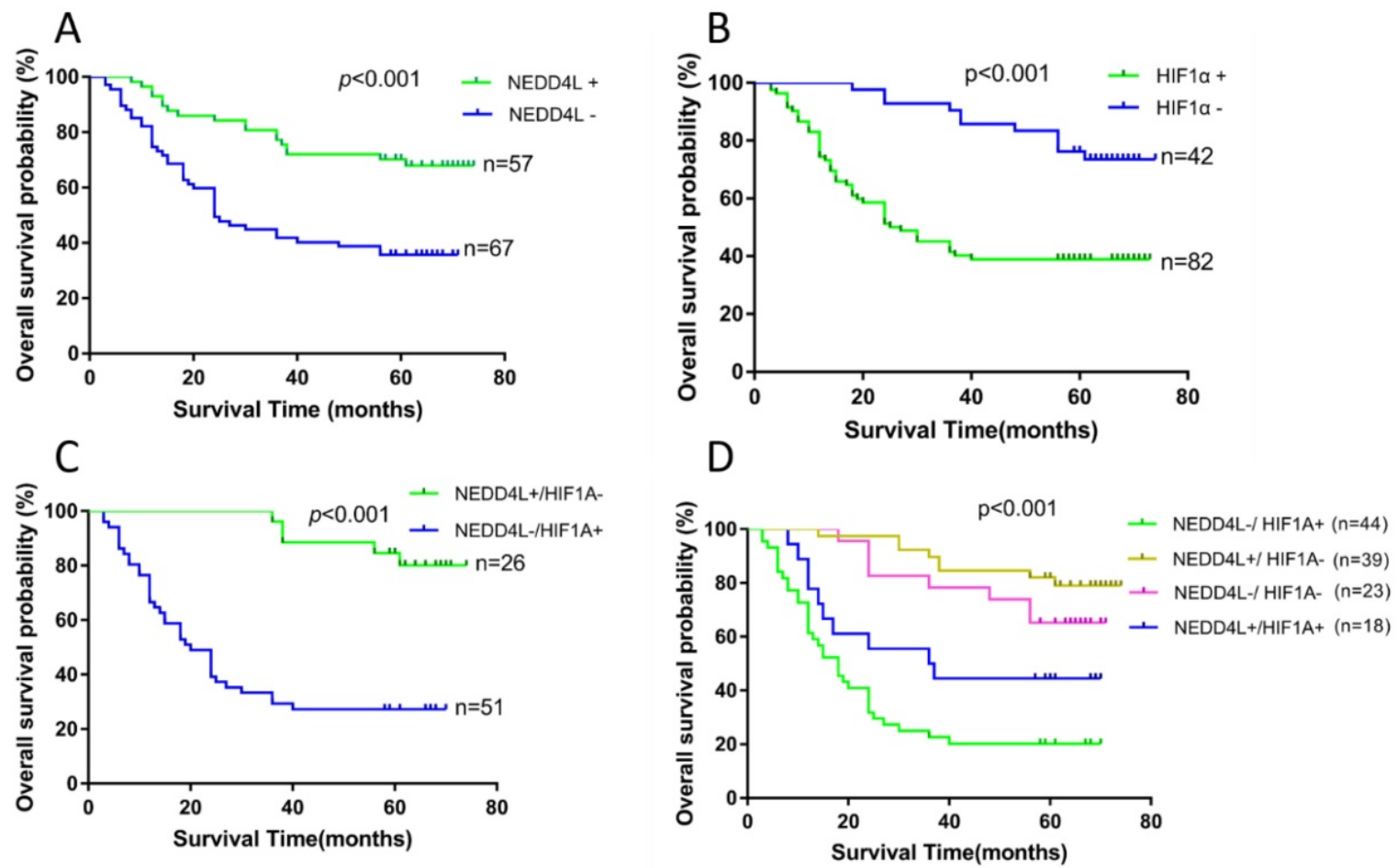

Figure 4. Kaplan-Meier survival analysis of the correlation between the combined NEDD4L and HIF-1 $\alpha$ expression and the OS of GC patients. (A): The OS of patients with NEDD4L - and NEDD4L +. (B): The OS of patients with HIFla+ and HIF-1 $\alpha$-. (C): Patients with NEDD4L + and HIF-1 $\alpha$ - were compared with the patients with NEDD4L -and HIF-1 $\alpha+.(D):$ The OS of patients with subgroups stratified by NEDD4L and HIF-1 $\alpha$ expression. 
Table 3. Univariate and multivariate analysis of the correlation between clinicopathological factors and prognosis

\begin{tabular}{|c|c|c|c|c|}
\hline \multirow[t]{2}{*}{ Variables } & Univariate analysis & \multirow[t]{2}{*}{$P$ value } & Multivariate analysis & \multirow[t]{2}{*}{$P$ value } \\
\hline & HR(95\%CI) & & HR(95\%CI) & \\
\hline Gender (male vs. female) & $0.782(0.407-1.503)$ & 0.46 & & NI \\
\hline Age (y) (<61 vs. $\geq 61)$ & $1.128(0.682-1.864)$ & 0.639 & & NI \\
\hline Tumor size $(\mathrm{cm})(<6$ vs. $\geq 6)$ & $0.544(0.326-0.905)$ & 0.019 & $1.140(0.670-1.939)$ & 0.63 \\
\hline Histological grade (Poor and other vs Well and moderate) & $3.425(1.557-7.535)$ & 0.002 & $2.715(1.224-6.023)$ & 0.014 \\
\hline Depth of invasion (T3/T4 vs T1/T2) & $11.393(2.779-46.7)$ & 0.001 & $2.674(0.393-18.209)$ & 0.315 \\
\hline Lymph node metastasis (yes vs. no) & $5.771(2.619-12.715)$ & 1.37E-05 & $2.539(0.200-32.213)$ & 0.472 \\
\hline TNM stages ( III/IV vs I/II.) & 7.987(3.779-16.880) & $5.26 \mathrm{E}-08$ & $2.282(0.165-31.455)$ & 0.583 \\
\hline NEDD4L expression (+ VS- ) & $0.354(0.204-0.615)$ & $2.30 \mathrm{E}-4$ & $0.494(0.279-0.875)$ & 0.016 \\
\hline HIF1aexpression(+ vs -) & $5.196(2.913-9.268)$ & 2.38E-8 & $4.606(2.450-8.661)$ & 2.12E-06 \\
\hline NEDD4L/HIF1a expression (NEDD4L-/HIF1a+ VS NEDD4L+/HIF1a-) & $0.121(0.055-0.266)$ & $1.36 \mathrm{E}-07$ & $0.140(0.059-0.333)$ & 4.42E-07 \\
\hline
\end{tabular}

Thus, our results indicate that NEDD4L and HIF-1a may be independent prognostic factors for GC. Concurrently, a more important prognostic indicator of GC patients may be the combination of NEDD4L and HIF-1a. Therefore, the mechanisms that NEDD4L- and HIF-1a- mediated regulation of GC progression need to be explored further, which may ultimately promote the development of new anticancer strategies.

\section{Acknowledgments}

We thank Xiao Song, Lingna Hu, Dapeng Yun, Yuqi Wang, Shiming Wang, Zhipeng Zhao, Shuangping $\mathrm{Ma}$, Hairui Xi, Chenqiang Jia, Chengde Zheng, Chao Wang, Qing Xiao, Zheng Fang, Youliang $\mathrm{Wu}$, Tao Zhang, Yuliang Zhou, Cheng $\mathrm{Wu}$, Ganbiao Wang for their technical supports. We thank Dr.Xiao Song for the helpful suggestions on this manuscript. We further thank all volunteers participated in this study.

\section{Funding}

This work was supported by grants from the National Natural Science Foundation of China (81672 389 and 81874063).

\section{Authors' Contributions}

Conceptualization, Xingwang Jiang. Shangxin Zhang and Yongxiang Li.; Methodology, Qiang Yan and Ruochuan Sun.; Formal analysis, Mingdian Lu and Yi Sheng.; Funding Acquisition, Yongxiang Li; Investigation, Zihuan Yin and Yi Sheng; Data Curation, Ruochuan Sun and Zhen Zhang; Resources, Xingwang Jiang, Shangxin Zhang, Zihuan Yin and Qiang Yan; Writing - Original Draft Preparation, Xingwang Jiang and Shangxin Zhang; Writing Review \& Editing, Xingwang Jiang and Yongxiang Li.; Project Administration, Yongxiang $\mathrm{Li}$ and Zhen Zhang.

\section{Competing Interests}

The authors have declared that no competing interest exists.

\section{References}

1. Soerjomataram I, Lortet-Tieulent J, Parkin DM, Ferlay J, Mathers C, Forman D, et al. Global burden of cancer in 2008: a systematic analysis of disability-adjusted life-years in 12 world regions. Lancet. 2012; 380: 1840-50.

2. Ferlay J, Steliarova-Foucher E, Lortet-Tieulent J, Rosso S, Coebergh JW, Comber $\mathrm{H}$, et al. Cancer incidence and mortality patterns in Europe: estimates for 40 countries in 2012. Eur J Cancer. 2013; 49: 1374-403.

3. $\mathrm{Hu} X Y, \mathrm{Xu} Y \mathrm{M}, \mathrm{Fu} \mathrm{Q}, \mathrm{Yu} J J$, Huang J. Nedd4L expression is downregulated in prostate cancer compared to benign prostatic hyperplasia. Eur J Surg Oncol. 2009; 35: 527-31.

4. Sakashita $\mathrm{H}$, Inoue $\mathrm{H}$, Akamine $\mathrm{S}$, Ishida $\mathrm{T}$, Inase $\mathrm{N}$, Shirao $\mathrm{K}$, et al Identification of the NEDD4L gene as a prognostic marker by integrated microarray analysis of copy number and gene expression profiling in non-small cell lung cancer. Ann Surg Oncol. 2013; 20 Suppl 3: S590-8.

5. Takeuchi T, Adachi Y, Nagayama T, Furihata M. Nedd4L modulates the transcription of metalloproteinase- 1 and -13 genes to increase the invasive activity of gallbladder cancer. Int J Exp Pathol. 2011; 92: 79-86.

6. Gao C, Pang L, Ren C, Ma T. Decreased expression of Nedd4L correlates with poor prognosis in gastric cancer patient. Med Oncol. 2012; 29: 1733-8.

7. He S, Deng J, Li G, Wang B, Cao Y, Tu Y. Down-regulation of Nedd4L is associated with the aggressive progression and worse prognosis of malignant glioma. Jpn J Clin Oncol. 2012; 42: 196-201.

8. Tanksley JP, Chen X, Coffey RJ. NEDD4L is downregulated in colorectal cancer and inhibits canonical WNT signaling. PloS one. 2013; 8: e81514.

9. Harvey KF, Kumar S. Nedd4-like proteins: an emerging family of ubiquitin-protein ligases implicated in diverse cellular functions. Trends Cell Biol. 1999; 9: 166-9.

10. Harvey KF, Dinudom A, Cook DI, Kumar S. The Nedd4-like protein KIAA0439 is a potential regulator of the epithelial sodium channel. J Biol Chem. 2001; 276: 8597-601.

11. Wang GL, Jiang BH, Rue EA, Semenza GL. Hypoxia-inducible factor 1 is a basic-helix-loop-helix-PAS heterodimer regulated by cellular O2 tension. Proceedings of the National Academy of Sciences of the United States of America. 1995; 92: 5510-4.

12. Denko NC. Hypoxia, HIF1 and glucose metabolism in the solid tumour. Nature reviews Cancer. 2008; 8: 705-13.

13. Schindl M, Schoppmann SF, Samonigg H, Hausmaninger $\mathrm{H}$, Kwasny W, Gnant $M$, et al. Overexpression of hypoxia-inducible factor 1alpha is associated with an unfavorable prognosis in lymph node-positive breast cancer. Clin Cancer Res. 2002; 8: 1831-7.

14. Birner $P$, Schindl $M$, Obermair A, Plank $C$, Breitenecker $G$ Oberhuber $G$. Overexpression of hypoxia-inducible factor 1alpha is a marker for an unfavorable prognosis in early-stage invasive cervical cancer. Cancer research. 2000; 60: 4693-6.

15. Aebersold DM, Burri P, Beer KT, Laissue J, Djonov V, Greiner RH, et al. Expression of hypoxia-inducible factor-1alpha: a novel predictive and prognostic parameter in the radiotherapy of oropharyngeal cancer. Cancer research 2001: 61: 2911-6.

16. Birner P, Schindl M, Obermair A, Breitenecker G, Oberhuber G. Expression of hypoxia-inducible factor 1alpha in epithelial ovarian tumors: its impact on prognosis and on response to chemotherapy. Clin Cancer Res. 2001; 7: 1661-8.

17. Lee BL, Kim WH, Jung J, Cho SJ, Park JW, Kim J, et al. A hypoxia-independent up-regulation of hypoxia-inducible factor-1 by AKT contributes to angiogenesis in human gastric cancer. Carcinogenesis. 2008; 29: 44-51.

18. Wu Y, Yun D, Zhao $Y$, Wang $Y$, Sun $R$, Yan $Q$, et al. Down regulation of RNA binding motif, single-stranded interacting protein 3 , along with up regulation of nuclear HIF1A correlates with poor prognosis in patients with gastric cancer. Oncotarget. 2017; 8: 1262-77.

19. Isobe $\mathrm{T}$, Aoyagi $\mathrm{K}$, Koufuji $\mathrm{K}$, Shirouzu K, Kawahara A, Taira T, et al. Clinicopathological significance of hypoxia-inducible factor-1 alpha (HIF-1alpha) expression in gastric cancer. Int J Clin Oncol. 2013; 18: 293-304. 
20. Urano N, Fujiwara Y, Doki Y, Tsujie M, Yamamoto H, Miyata H, et al. Overexpression of hypoxia-inducible factor-1 alpha in gastric adenocarcinoma. Gastric Cancer. 2006; 9: 44-9.

21. Wang Z, Dang T, Liu T, Chen S, Li L, Huang S, et al. NEDD4L Protein Catalyzes Ubiquitination of PIK3CA Protein and Regulates PI3K-AKT Signaling. J Biol Chem. 2016; 291: 17467-77.

22. Russo CJ, Melista E, Cui J, DeStefano AL, Bakris GL, Manolis AJ, et al. Association of NEDD4L ubiquitin ligase with essential hypertension. Hypertension. 2005; 46: 488-91.

23. Kito Y, Bai J, Goto N, Okubo H, Adachi Y, Nagayama T, et al. Pathobiological properties of the ubiquitin ligase Nedd4L in melanoma. Int J Exp Pathol. 2014; 95: $24-8$.

24. Kuratomi G, Komuro A, Goto K, Shinozaki M, Miyazawa K, Miyazono K, et al. NEDD4-2 (neural precursor cell expressed, developmentally down-regulated 4-2) negatively regulates TGF-beta (transforming growth factor-beta) signalling by inducing ubiquitin-mediated degradation of Smad2 and TGF-beta type I receptor. Biochem J. 2005; 386: 461-70.

25. Gao S, Alarcon C, Sapkota G, Rahman S, Chen PY, Goerner N, et al. Ubiquitin ligase Nedd4L targets activated Smad2/3 to limit TGF-beta signaling. Mol Cell. 2009; 36: 457-68

26. Ding $Y$, Zhang $Y, X u$ C, Tao QH, Chen YG. HECT domain-containing E3 ubiquitin ligase NEDD4L negatively regulates Wnt signaling by targeting dishevelled for proteasomal degradation. J Biol Chem. 2013; 288: 8289-98.

27. Vaupel P, Kallinowski F, Okunieff P. Blood flow, oxygen and nutrient supply, and metabolic microenvironment of human tumors: a review. Cancer research. 1989; 49: 6449-65.

28. Zhong H, De Marzo AM, Laughner E, Lim M, Hilton DA, Zagzag D, et al. Overexpression of hypoxia-inducible factor 1alpha in common human cancers and their metastases. Cancer research. 1999; 59: 5830-5.

29. Dewhirst MW, Cao Y, Moeller B. Cycling hypoxia and free radicals regulate angiogenesis and radiotherapy response. Nature reviews Cancer. 2008; 8: $425-37$. 